\title{
Design, fabrication, and testing of stellar coronagraphs for exoplanet imaging
}

Justin M. Knight, John Brewer, Ryan Hamilton, Karen Ward, Tom D. Milster, et al.

Justin M. Knight, John Brewer, Ryan Hamilton, Karen Ward, Tom D. Milster, Olivier Guyon, "Design, fabrication, and testing of stellar coronagraphs for exoplanet imaging," Proc. SPIE 10400, Techniques and Instrumentation for Detection of Exoplanets VIII, 104000N (12 September 2017); doi: $10.1117 / 12.2273558$

EviE Event: SPIE Optical Engineering + Applications, 2017, San Diego, California, United States 


\title{
Design, fabrication, and testing of stellar coronagraphs for exoplanet imaging
}

\author{
Justin M. Knighta,b, John Brewer ${ }^{\mathrm{a}}$, Ryan Hamilton ${ }^{\mathrm{a}}$, Karen Ward $^{\mathrm{a}}$, Thomas D. Milster ${ }^{\mathrm{a}}$, and \\ Olivier Guyon ${ }^{\mathrm{a}, \mathrm{b}, \mathrm{c}}$ \\ aThe College of Optical Sciences, University of Arizona, 1630 E. University Blvd., Tucson, AZ \\ 85719, United States \\ bsteward Observatory, University of Arizona, 933 N. Cherry Ave., Tucson, AZ 85719, United \\ States

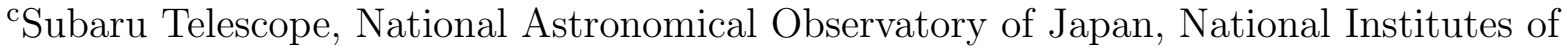 \\ Natural Sciences, 650 N. A'ohoku Place, Hilo, HI 96720, United States
}

\begin{abstract}
Complex-mask coronagraphs destructively interfere unwanted starlight with itself to enable direct imaging of exoplanets. This is accomplished using a focal plane mask (FPM); a FPM can be a simple occulter mask, or in the case of a complex-mask, is a multi-zoned device designed to phase-shift starlight over multiple wavelengths to create a deep achromatic null in the stellar point spread function. Creating these masks requires microfabrication techniques, yet many such methods remain largely unexplored in this context. We explore methods of fabrication of complex FPMs for a Phased-Induced Amplitude Apodization Complex-Mask Coronagraph (PIAACMC). Previous FPM fabrication efforts for PIAACMC have concentrated on mask manufacturability while modeling science yield, as well as assessing broadband wavelength operation. Moreover current fabrication efforts are concentrated on assessing coronagraph performance given a single approach. We present FPMs fabricated using several process paths, including deep reactive ion etching and focused ion beam etching using a silicon substrate. The characteristic size of the mask features is $5 \mu \mathrm{m}$ with depths ranging over $1 \mu \mathrm{m}$. The masks are characterized for manufacturing quality using an optical interferometer and a scanning electron microscope. Initial testing is performed at the Subaru Extreme Adaptive Optics testbed, providing a baseline for future experiments to determine and improve coronagraph performance within fabrication tolerances.
\end{abstract}

Keywords: stellar coronagraph, focal plane mask, complex FPM, PIAACMC, microfabrication, exoplanet imaging

\section{INTRODUCTION}

Stellar coronagraphs are telescope instruments designed to suppress starlight to image nearby, faint planetary companions, or exoplanets. This task is challenging, as the coronagraph must yield a star-planet contrast ratio of several orders of magnitude at a very small angular separation. However, it is well worth achieving, as it can enable spectroscopic characterization of exoplanets; this permits study of previously unavailable information such as atmospheric composition, allowing one to characterize the habitability of the exoplanet under observation. ${ }^{1}$

Coronagraph architectures can be divided into external and internal occulters, which vary in degree of difficulty to design, fabricate, and test. We focus on a subset of internal occulters which marry multiple coronagraphic components into one design to yield high contrast at a small inner-working angle (IWA), known as complex-mask coronagraphs (CMC). Guyon et. al introduces the concept of a complex focal plane mask (FPM) to improve the performance of previously developed coronagraphs: the Apodized Pupil Lyot Coronagraph (APLC) and the Phase-Induced Amplitude Apodization Coronagraph (PIAAC). ${ }^{2}$ With the addition of the complex FPM, the corresponding performance metrics, e.g. the contrast, IWA, and the throughput are enhanced by comparison with their ideal FPM counterparts. The two CMC architectures presented are referred to as the Apodized Pupil

Further author information: (Send correspondence to Justin M. Knight)

E-mail: jknight@optics.arizona.edu

Techniques and Instrumentation for Detection of Exoplanets VIII, edited by Stuart Shaklan, Proc. of SPIE Vol. 10400, 104000N · (C) 2017 SPIE · CCC code: 0277-786X/17/\$18 · doi: 10.1117/12.2273558 
Complex-Mask Lyot Coronagraph (APCMLC) and the Phase-Induced Amplitude Apodization Complex-Mask Coronagraph (PIAACMC). In both cases the ideal FPM - typically an opaque spot occulting the entirety of the stellar point spread function (PSF), is reduced in size and split into a number of subregions, or zones, over which the optical path length delay can be changed independently of any other zone. These complex FPMs are responsible for providing stellar suppression in broadband light over the core of the stellar PSF: the zones delay local patches of incoming wavefront such that destructive interference occurs in the stellar PSF up to a $20 \%$ bandwidth. $^{3}$ Additionally, complex FPMs offer some resilience to the effects of stellar angular size, but are notably sensitive to tip-tilt errors and other low-order aberrations because of their aggressive IWA. In the case of PIAACMC, complex FPMs offer a trade-off in fabrication: the PIAA optics become less aspheric and thus easier to fabricate at the cost of producing a micro-scale device with a continuous range of depths.

While there are several advantages to implementing complex FPMs, they remain relatively unexplored in laboratory settings. The NASA Ames Coronagraph Experiment (ACE) testbed has demonstrated a fabricated complex FPM, reaching $9.09 \cdot 10^{-6}$ average contrast over a $10 \%$ bandwidth centered at $580 \mathrm{~nm} .{ }^{4}$ Moreover the effects of fabrication errors have only been modeled for impact on coronagraph performance on the WFIRSTAFTA mission; in the presence of fabrication errors, a modeled PIAACMC achieved the WFIRST-AFTA Study milestone 3 raw contrast of $10^{-8}$ for a $10 \%$ band at $550 \mathrm{~nm}^{5}$ Generally speaking, it is also unknown which of the many fabrication processes should be used to produce complex FPMs. In this paper we explore the field of microfabrication techniques for possible process paths to create complex FPMs, along with demonstrating fabricated complex FPM performance using a PIAACMC at the Subaru Extreme Adaptive Optics (SCExAO) testbed. ${ }^{6}$

We subscribe to the production workflow shown in Figure 1 to fabricate complex FPMs. This workflow demonstrates a fabrication cycle for a particular process path given a set of requirements. The requirements are derived from the optical design parameters of the coronagraph architecture and testing facilities; these include wavelength range operation, use in transmission or reflection, and the type of optics in the focal plane, e.g. passive or active devices. The coronagraph design process yields ideal optical elements which must be mapped to a manufacturable state to attempt a fabrication process. This entails folding the optical requirements, available process equipment and expertise together to create a viable process path which closely approximates the ideal design.

After fabrication the production workflow encompasses device characterization and test preparation. The information collected from measurement equipment can be used to simulate the performance of the coronagraph more accurately by representing effects from fabrication errors and antireflection (AR) coatings. The final stage in the cycle is testing. It provides scientific benchmarks for coronagraph performance while also relaying feedback for a more optimal coronagraph design. This can be interpreted as a design which is easier to fabricate or performs better. Ideally a more optimal design will achieve both.

In Section 2 we briefly describe the design process and detail its relationship with fabrication. A CMC design prescription is introduced for the PIAACMC at SCExAO focusing on complex FPM manufacturability for a selected fabrication process. The ideal coronagraph performance is briefly reviewed and discussed. Section 3 summarizes the fabrication cycles and process path exploration by characterizing fabricated devices. Section 4 demonstrates the test preparation for the complex FPMs and details their installation and first light at SCExAO. In Section 5 we discuss future considerations for fabrication cycles as well as experiments to determine and optimize the complex FPM performance and assess performance limitations due to fabrication tolerances.

\section{CMC DESIGN FOR FABRICATION}

An ideal CMC design for fabrication is one which can be used successfully, reliably and repeatably to produce functioning complex FPMs. To achieve this, we choose a design robust against anticipated sources of error such as lateral misalignments and depth tolerances, yet considered manufacturable using typical fabrication equipment common in a research cleanroom environment. Ideally, the resulting manufacturable complex FPM should be fabricated to the specified tolerances set by the design as well as the process equipment. This suggests the tolerances of the design should reflect the process equipment limitations. For our workflow, this is a work in progress accounted for by choosing design parameters that are a priori manufacturable.

Proc. of SPIE Vol. 10400 104000N-2 


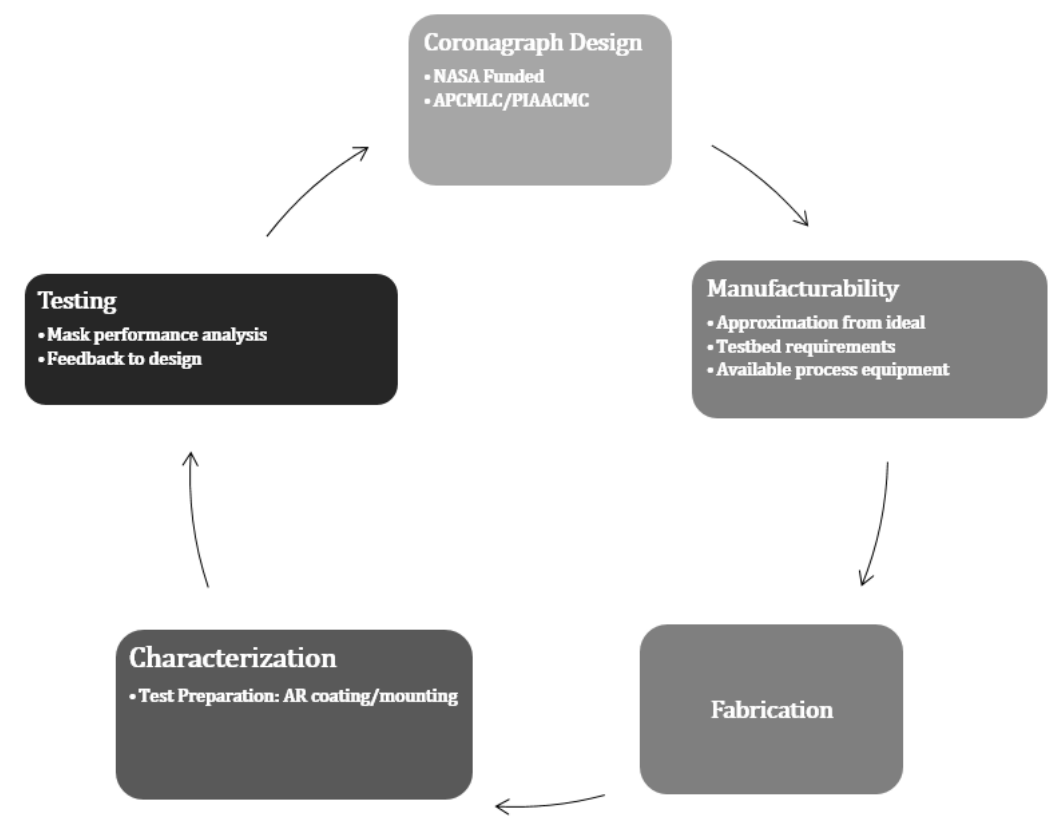

Figure 1. The charted workflow adopted for exploring fabrication methods of complex FPMs.

\subsection{Design Process}

The design process begins by describing the optical layout and parameters of a system. PIAACMCdesign, the open source software developed in C for producing APCMLC and PIAACMC designs by Olivier Guyon, ${ }^{7}$ uses the optical description of a system to generate a coronagraph solution for a monochromatic, on-axis point source assuming an ideal FPM. The assumptions of monochromaticity and an unresolved light source are then removed to produce a more robust and realistic solution using a complex FPM. Breaking these assumptions requires an iterative optimization routine to find a complex FPM solution capable of producing the desired performance metrics. An additional set of parameters for the complex FPM can be given to address some fabrication concerns. Namely the size (or number) and shape of FPM zones, as well as their corresponding depths can be controlled to steer the optimization routine to complex FPM solutions which can be considered manufacturable.

\subsection{Fabrication Processes}

For a passive complex FPM, that is, one which interacts with light through a fixed physical pattern as is typical with most masking optics, there are two fabrication paths. These are additive microfabrication device manufacturing, in which material is deposited to a substrate surface to produce a pattern, or subtractive microfabrication device manufacturing, in which the substrate material is etched away, leaving a pattern. Generally speaking, additive or subtractive manufacturing can be performed in grayscale or binary, the latter being easier to accomplish. The previous complex FPM example at the ACE testbed was created using a standard binary subtractive process, whereas the WFIRST-AFTA complex FPM was fabricated using a grayscale additive process. Our experience and available equipment strongly encourages binary subtractive processing for two reasons. The first reason is the robustness against device failure given by the ability to have multiple devices on a single substrate. The second reason is the capability to rapidly iterate on the design process once the process is matured.

When considering optical systems for coronagraph testing, transmissive complex FPMs are required. This does not preclude us from fabricating devices which may not work in transmission, but are successfully built to 
design tolerances. This is what we refer to as process exploration, and encompasses the majority of our work outside of the main process path of binary subtractive processing.

\subsection{Selected Design}

Given our available process equipment of photomask exposure tools and reactive ion etchers, the choice to include coronagraph level testing introduces the need for the SCExAO testbed. This is a trade-off between our experience and successfully fabricating a complex FPM by some available process. The substrate most suitable for successful, reliable, and repeatable production is the semiconductor industry standard silicon. We use the process of binary optics, in which the depths are discretized and characterized by levels of $2^{n}$, where $\mathrm{n}$ is the number of etch steps. ${ }^{8}$ This is a binary subtractive process with uniformly spaced depths.

\subsubsection{Complex FPM}

The complex FPM from the SCExAO PIAACMC design is depicted in Figure 2(a). This multi-zone FPM consists of 32 concentric rings of hexagons, each of which is $4.55 \mu \mathrm{m}$ in side-length. The tip-to-tip diameter of each hexagon is $9.10 \mu \mathrm{m}$. Hexagons are chosen to provide better tolerance to edge effects compared to ring sectors from previous designs such as the WFIRST-AFTA complex FPM. ${ }^{5}$ Features several microns in size are standard in microfabrication; the size of the hexagons is chosen to reflect this fact while also reducing the effects of fabrication errors from line-on-line mis-registrations between etch steps. The continuous range of depths spans $0-1 \mu \mathrm{m}$. The spatial extent of the mask is approximately $295 \mu \mathrm{m}$ across, covering a total of six $\lambda / \mathrm{D}$ in H-band. Figure 2(b) demonstrates the 16 level approximation to the ideal complex FPM suitable for fabrication. The unit etch step depth is $60.3 \mathrm{~nm}$ at 16 levels; this is strongly approaching the limit of accurate etching without active feedback during the etch process.

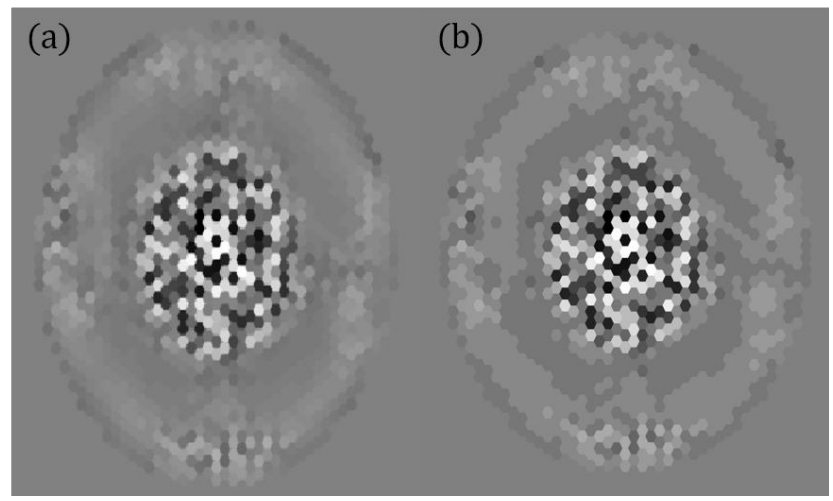

Figure 2. (a) An ideal complex FPM consisting of 1237 hexagons over a continuous range of depths. This is one of many possible computed solutions, as it potentially reflects a local minima. (b) An approximate design to the ideal complex FPM in (a) features 16 discretized depths spaced by $60.3 \mathrm{~nm}$ for a total range of 0 to $900 \mathrm{~nm}$.

Figure 3 depicts the process to fabricate the approximate complex FPM design. The 16 level approximation is encoded by patterning hexagons in photoresist which are relieved, or etched, from the surface of the silicon substrate using a dry etching process. For a total of 16 levels, there are four required etch steps. The complex FPM perform better with positive and negative depth features. Thus an additional etch step is required to achieve a new zero reference for the complex FPM. To achieve this bipolarity, the entire surface around the complex FPM must be etched to half of the total feature depth. In our scheme, this is encoded as a separate photolithographic-etch step known as the bipolar step.

\subsubsection{Binary Fabrication Cycles}

Two fabrication cycles of this complex FPM were performed using silicon wafers. The patterning process for our first fabrication cycle used a 3in. silicon wafer coated with photoresist. A photomask, or transparent glass plate with light absorbing chrome to represent the hexagonal patterns in Figure 3, was created which contains 15 exposure patterns for each of the four etch steps. An additional photomask was used to produce the bipolar 


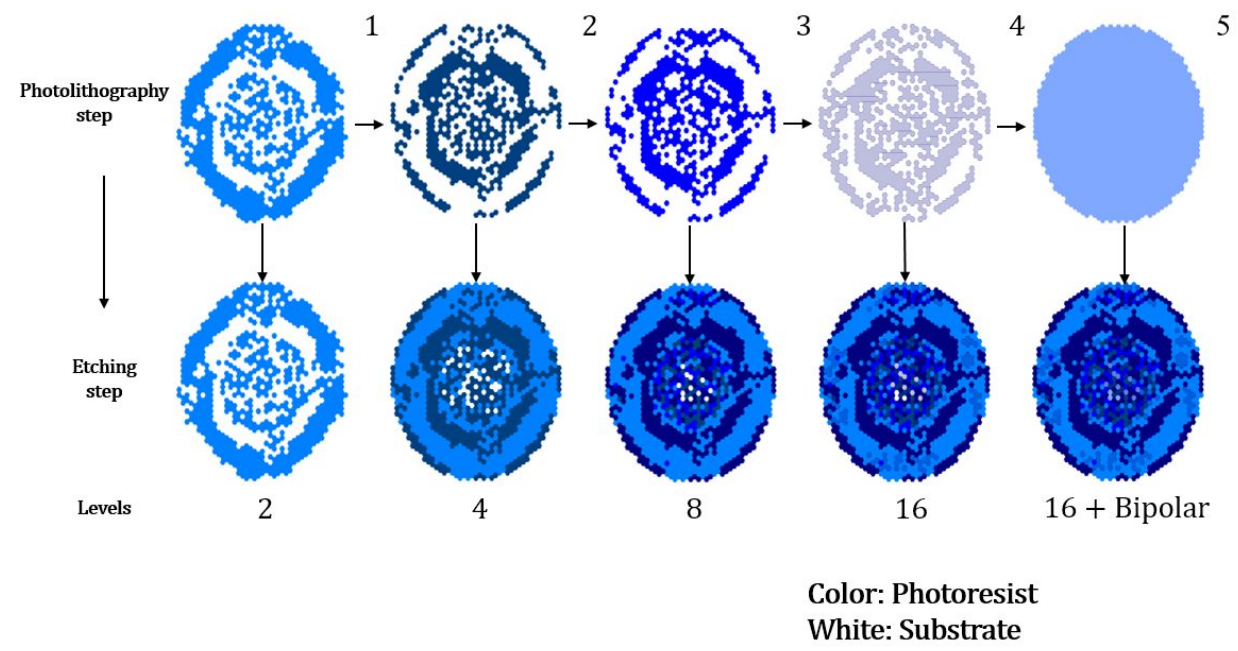

Figure 3. The binary optics scheme for fabrication of the SCExAO PIAACMC complex-mask. In the top row, photoresist is exposed to light shining through a pattern of hexagons and developed, leaving a mixture of substrate (white) and photoresist (color) to be etched. At each corresponding etch step in the bottom row, the pattern is written into the substrate, creating a total of $2^{n}$ discrete depths. An additional bipolar etch is required to change the zero reference point from the top of the substrate to the middle depth.

pattern. Similarly, an optically reduced photomask, referred to as a reticle ${ }^{9}$ was used to create an array of 169 complex FPMs patterns per process step on a 4in. silicon wafer after applying photoresist.

The main difference between the two fabrication cycles is which etch method was performed. In the case of the 3in. wafer, a deep silicon etch known as the Bosch process was performed to achieve vertical sidewall features with high selectivity, or etch rate ratios, between the photoresist and silicon. ${ }^{9}$ Meantime, the 4in. wafer was etched using an reactive ion etcher with inductively coupled plasma for increased anisotropic etching. This has worse selectivity, but its etch rate can be controlled more accurately than the Bosch process for depths which require nanometer scale precision.

Tolerancing of each fabrication cycle requires simulations to determine which variables are the most damaging to coronagraph performance. As a first effort, we used the highest precision of lateral tolerances available for the equipment: approximately $200 \mathrm{~nm}$ repeatable feature alignment in both fabrication cycles. The etch depth tolerances were computed for the ideal case as three standard deviations from the shallowest etch depth of 60.3 $\mathrm{nm}$ in each direction, or $\pm 15 \mathrm{~nm}$. This is a difficult tolerance to meet using subtractive manufacturing, and one which may be able to be relaxed through simulation investigations previously mentioned.

\subsection{Ideal Performance}

The PIAACMC for SCExAO is designed for $80 \%$ throughput with a $1.2 \lambda / \mathrm{D}$ IWA over H-band using the Subaru pupil without bad DM actuator masking features. The throughput is measured as the ratio of residual starlight in the PSF at an angular separation to the amount of starlight passing through without any suppression at that same point. Contrast curves for this design are depicted in Figure 4 at three increasing stellar angular sizes in $\mathrm{H}$-band. The curves reflect the raw contrast computed at each angular separation in units of $\lambda / \mathrm{D}$. The missing values at small IWA are a design feature; typically these values are interpolated. This is a high performance coronagraph, but these curves are not representative of how well this coronagraph will perform with fabricated components. The main limitations will be due to fabrication errors and sensitivity to low-order aberrations, of which wavefront sensing and control can help mitigate. 


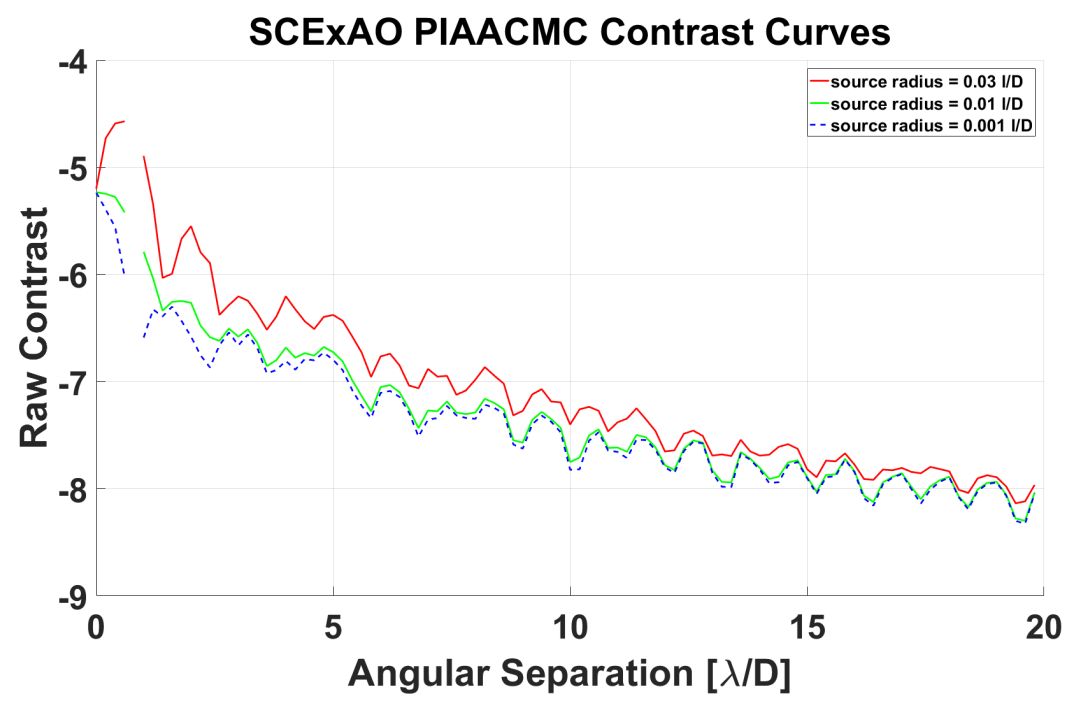

Figure 4. Raw contrast curves for a high performance PIAACMC at SCExAO. Contrast reduces as a function of stellar angular size and angular separation.

\section{FABRICATION RESULTS}

We present an overview of the results from the fabrication process cycles and process exploration. The completed complex FPMs are separate from the process exploration efforts, which resulted in partially fabricated devices open to further development.

After fabrication both optical interferometer and scanning electron microscope (SEM) images were taken of the finished complex FPMs. The final complex FPMs have 16 distinct levels exhibiting fabrication errors in both lateral dimensions and depth as shown in Figure 5(b) and (c). Figure 5(a) is the 16 depth approximation in matching grayscale for reference. The lateral misalignments and mis-registration of hexagons between etch depths produce unwanted diffraction effects which have yet to be accounted for in design work.
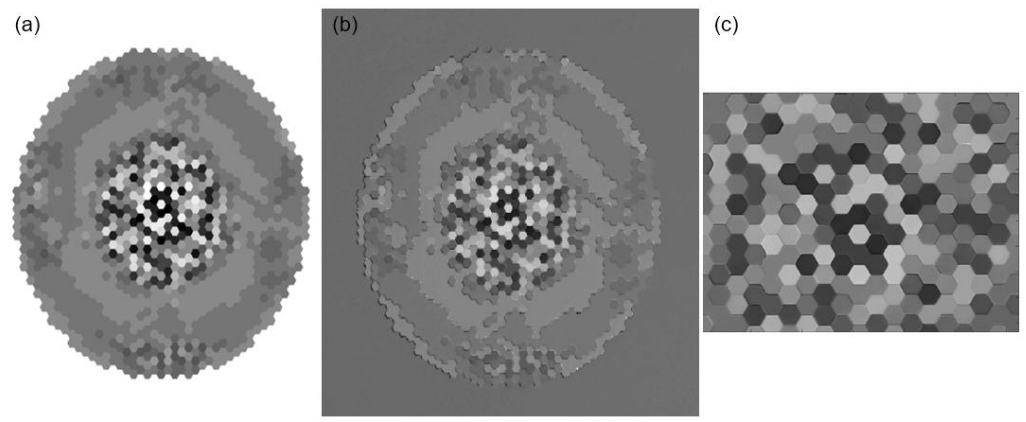

Figure 5. (a) The 16 level approximation to the ideal complex FPM design. (b) A complex FPM after a fabrication cycle. (c) $0.2 \mu \mathrm{m}$ sampling across the modulated central region of the complex FPM from (b). This demonstrates some of the misalignments and their subsequent effects on depth uniformity around the hexagon edges.

The example measurement depths (red) in Figure 6 compare closely with the actual prescribed depths (blue). They demonstrate some lingering issues with etching multiple steps without active feedback. Namely the smaller the etch depth required, the harder it is to reach the depth without accurate measurement tools providing feedback. Moreover Figure 6 displays the limitations for using only an optical interferometer. Each time a steep 
slope occurs, there are numerical artifacts from poor sampling of those transition regions. A higher sampling measurement using a tool such as an atomic force microscope (AFM) would reveal the transition regions in adequate detail to provide meaningful information for their potential effects on coronagraph performance.

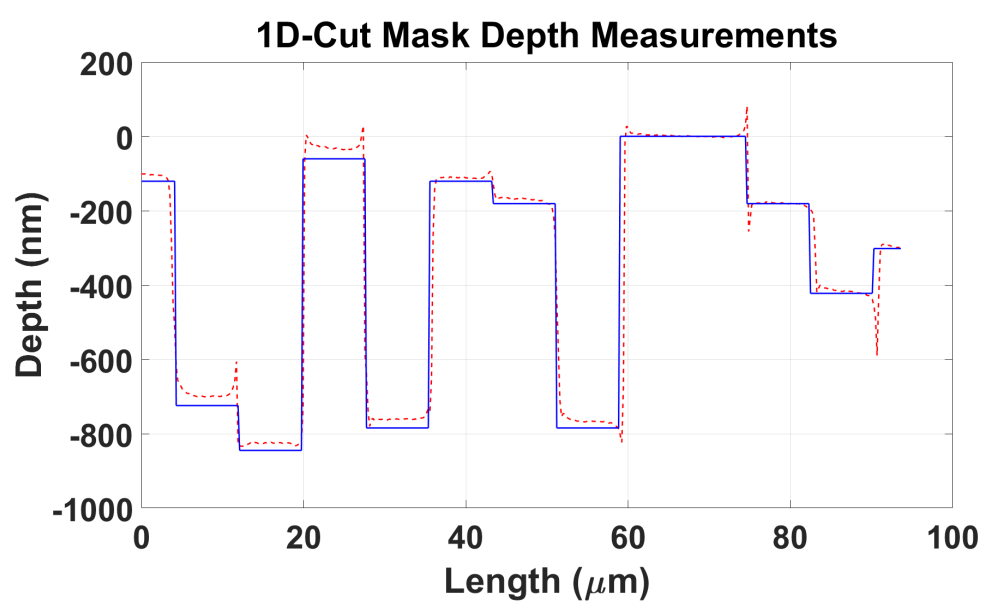

Figure 6. The depth targets (blue) show 12 distinct levels along this particular cut of Figure 5(c). The actual depths (red) follow closely along the depth targets, except for the shallowest depth. This is where the measurement tools monitoring the etch rate were reaching their accuracy limit.

Figure 7(a) and (b) depict the difficulties of line-on-line process work. This is always challenging to do in microfabrication because photoresists have graded slopes which transfer to the substrate material upon etching. This has the effect of biasing the size of the hexagons by creating a smoother transition region than the abrupt boundaries of the approximate design. However, an ideal complex FPM transitions between regions more closely to the fabricated version. Nevertheless, these transition regions must be studied and quantified to accurately predict complex FPM performance. Figure 7(c) and (d) show some examples of $200 \mathrm{~nm}$ photolithographic precision between process steps. In both examples the result is the same. There are edge features which are unaccounted for in the design and must be simulated to determine their effects.

We also note that at small scales, defined on the order of the observation wavelength, modeling fabrication errors requires more sophisticated strategies involving electric field modeling and propagation instead of the standard Fresnel propagator model. In addition, to more accurately determine depths with an SEM, we can cleave complex FPM samples. While the depth measurements are extremely accurate, this is a destructive and time consuming measurement to make which may be performed adequately enough using an AFM.

\subsection{Process Exploration}

Process exploration was performed using several different process equipment including a focused ion beam (FIB) tool, e-beam lithography (EBL) tool, and Nanoscribe 3D printer at the University of Arizona. We discuss the results of the FIB and note that more exploration is required for both the EBL and Nanoscribe process paths. In the case of the EBL, this is a matter of developing the appropriate process for fabricating a complex FPM. High resolution patterning is gained at the cost of write speed, hence device duplication. If high resolution features are not required, there may be no need to use the slow write time of the EBL. For the Nanoscribe 3D printer, a resolution of $500 \mathrm{~nm}$ is simply not good enough for the SCExAO complex FPM in the depth dimension. The result was missing hexagons in the pattern which were shallower than this depth. Moreover there are many outstanding issues such as field stitching and photoresist development to overcome before a complex FPM is produced with this method.

FIB etching is commonly used for photomask feature correction. In this context, it can be used as a corrective tool for missed depth targets assuming a shallow etch. Figure 8(a) and (b) demonstrates this for a small region of hexagons on a complex FPM sample. With a $30 \mathrm{kV}$ beam the corrective etch is uniform, but at the cost of depositing Gallium ions into the silicon surface $25 \mathrm{~nm}$ deep. Lowering the beam to $5 \mathrm{kV}$ as shown in Figure 8(c) 


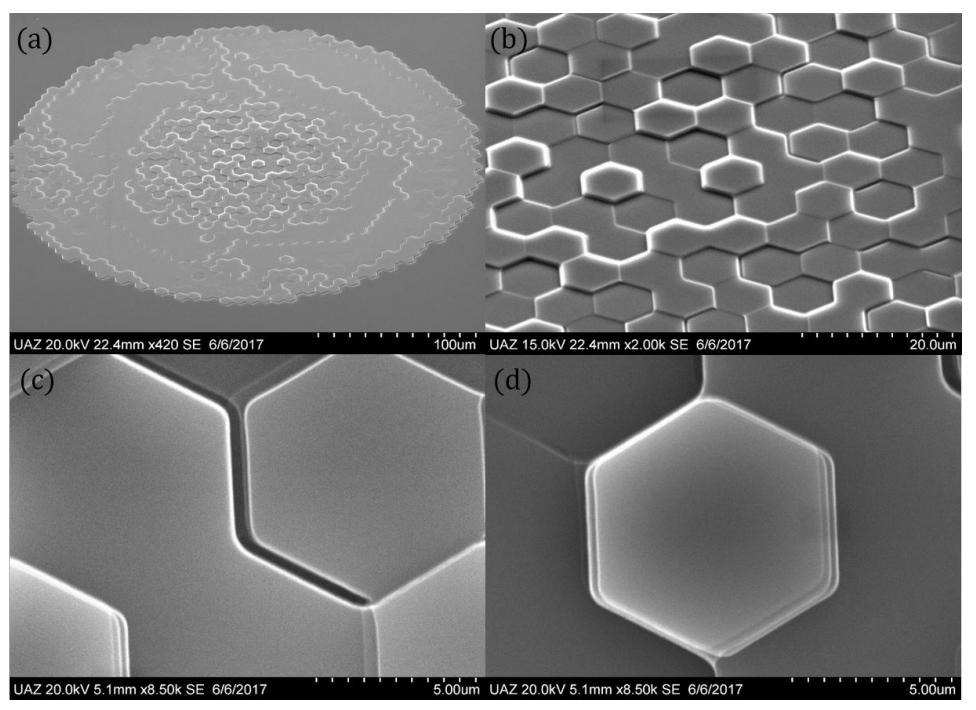

Figure 7. (a) SEM image at a 60 degree tilt of an entire complex FPM. (b) Large scale demonstration of line-on-line mis-registrations between process steps. (c) A $500 \mathrm{~nm}$ gap between levels creates an edge feature which may better modeling to see its effect on the mask's performance. (d) Misalignment between multiple levels effectively increases the size of this hexagon creating non-ideal edge effects.

and (d) reduces the Gallium ion implantation to around nine $\mathrm{nm}$ in this sample, but the etch is now unevenly distributed across the surface of the hexagon.

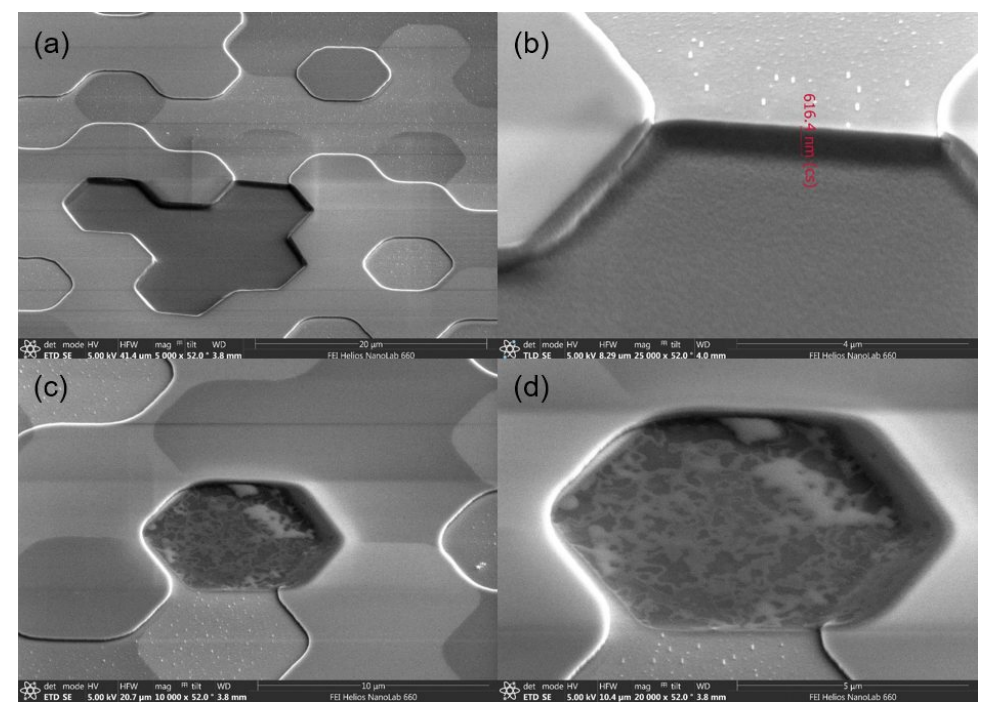

Figure 8. (a) $30 \mathrm{kV}$ beam etching into the surface of five hexagons. (b) The etch is uniform, but Gallium ions deposit into the silicon surface. (c) A $5 \mathrm{kV}$ beam is used to etch a single hexagon with noticeable effects. (d) The etch uniformity of the FIB is compromised to reduce the Gallium ion deposition to nine nm into the silicon surface.

If a design accounts for the Gallium ion implantation, or the effects are concluded to be negligible, there is potential for the FIB to be used as a corrective tool over sub-regions of a complex FPM. More characterization of this process is required to determine its usefulness. 


\section{TEST PREPARATION AND FIRST LIGHT}

The fabrication process generates devices which, when characterized, provide an estimate to their success at meeting design performance metrics in simulation. Prior to any testing of the devices, they must be prepared for installation. When creating devices using wafers, this typically entails dicing, coating, and mounting individual masks from the wafer batch.

\subsection{Dicing, Coating, and Mounting}

Processed wafers often contain multiple devices for a given fabrication cycle. The 4in. and 3in. silicon wafers used to fabricate the complex FPMs contained 169 and 15 potentially useful devices respectively. Each of the wafers was sent to a company with dicing specifications to match the $\mathrm{x}-\mathrm{y}$ translation limits over a 1in. diameter focal plane mount at SCExAO such that up to nine complex FPMs could fit. After dicing, select complex FPM pieces were commercially coated using a proprietary antireflective (AR) coating over H-band.

The AR coating reflectivity curve is shown in Figure 9. It measures wavelength against percent reflectivity, with a minimum reflectivity of about $0.3 \%$ at the central wavelength and an average reflectivity over H-band of less than $2 \%$. These coatings are necessary for use in the near-IR with silicon to avoid spurious back-reflections from stray light. The AR coating is applied to both sides of the complex FPMs using an ebeam evaporator. This instrument can deposit thin films anisotropically so that the bottom surfaces of the hexagons are coated while the sidewalls are largely free of any significant material deposition. This is done to provide a uniform coating across all hexagon depths, i.e. the areas where, if left uncoated, spurious light can have a potentially significant impact on the destructive interference effect of the complex FPM.

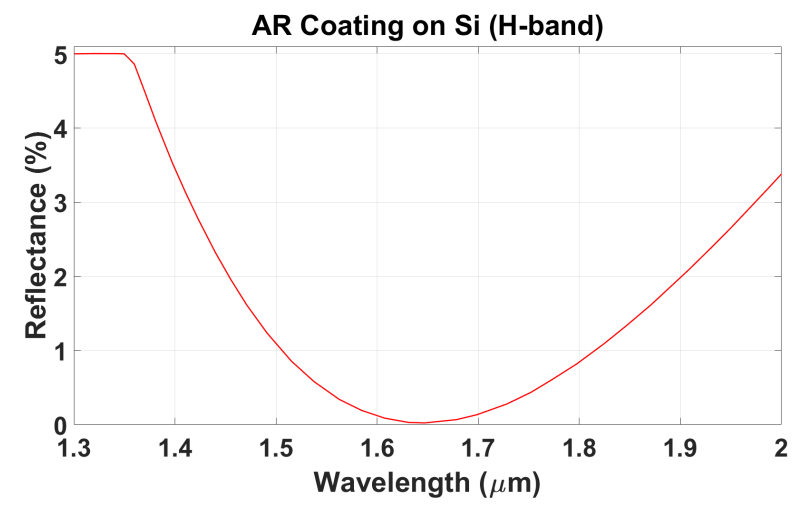

Figure 9. The antireflective coating applied to the complex FPMs determined to be in spec. This coating is a stack of thin film layers designed to provide low reflectivity over H-band. The average reflectivity over the entire curve is about $2 \%$.

After deposition of the AR coating, the complex FPMs were mounted at SCExAO using a 3D printed plastic resin adapter plate; it is custom designed to mechanically constrain arrays of complex FPMs to a plane. Figure 10(a) shows 18 complex FPMs in adapter plates mounted at SCExAO.

\subsection{First Light}

Upon installation at SCExAO, one of the complex FPMs was tested to determine how well it worked without any parameter tuning. Figure 10(b) depicts a pupil plane image of the effect of the complex FPM. To first order, the complex FPM produces an achromatic $\pi$ phase-shift over the core of the stellar PSF. Light from the stellar PSF is diffracted into the central obscuration and spiders, as well as outside the geometric pupil. There is some residual light left in the pupil from various effects including fabrication errors and spider features not included in the SCExAO PIAACMC design, but most notably a lack of PIAA optics aligned in the system. 
(a)

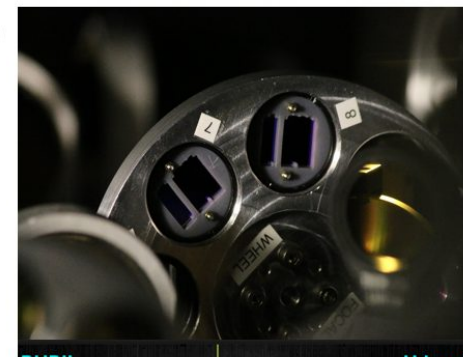

(b)

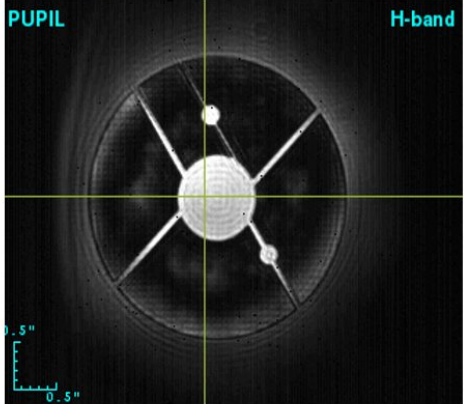

Figure 10. (a) The mounted complex FPMs. A total of 18 complex FPMs across two adapter plates allows for testing redundancy as well as determining which may of the wafer batches may function best. (b) An image of the pupil plane after the complex FPM demonstrates a functional device, but with room for improvement.

\section{CONCLUSIONS AND FUTURE WORK}

We fabricated and characterized complex FPMs designed for a high-performance PIAACMC at SCExAO. We attempted several fabrication methods with varying degrees of success. The main process path of photolithographic patterning and reactive ion etching produced two fabrication cycles of masks using the same coronagraph design. Each cycle yielded masks within the specified depth tolerances, but there are outstanding fabrication issues which can be either mitigated or resolved through process calibration and maturation. These issues can be addressed by introducing photomask biasing as well as other forms of process optimization considering photoresists and etch chemistries. Alternatively, an entirely new process can be developed as new process equipment becomes available which may be better equipped to achieve required tolerances. After fabrication the complex FPMs were characterized using an optical interferometer and scanning electron microscope. These detail the issues of lateral misalignments between process steps, as well as depth non-uniformities and some edge effects. The use of other characterization equipment such as an AFM will reveal detailed information in the transition regions between hexagons. When coupled together, each of these measurements will provide a model of the complex FPM which, when used in simulation, demonstrates the expected coronagraph performance given the limitations imposed by fabrication errors.

First light through the successfully fabricated complex FPMs demonstrates their ability to optically induce destructive interference over multiple wavelengths simultaneously. Telescope, camera, and wavefront control experiments will be performed at SCExAO to determine the optimal configuration for starlight suppression. Several performance metrics including contrast, throughput, and IWA will be measured in order to demonstrate the current limitations of the fabricated complex FPMs. Moreover PIAA optics will be aligned with the complex FPM to test a PIAACMC configuration using the available broadband test source. Doing so will be the first step toward determining the science yield of this coronagraph architecture. Furthermore, on-sky testing will be crucial to demonstrating the technological maturation of PIAACMC. Comparing performance limitations due to the complex FPMs with simulation will require an assessment of the testbed with and without the complex FPMs. A Zernike wavefront sensor ${ }^{10}$ can be used to reduce non-common path aberrations in the system, allowing for a more accurate assessment of the effects of the complex FPM fabrication errors. 


\section{ACKNOWLEDGMENTS}

This research is funded by TRIF optics and the NASA ExEP SCDA Study. A portion of this work was done in the UCSB Nanofabrication Facility, an open-access laboratory. A portion of this work was done in the ASU Nanofab Facility.

\section{REFERENCES}

[1] Guyon, O., Kern, B., Rodack, A., Knight, J., Belikov, R., Sirbu, D., Bryson, S., Henze, C., Codona, J., and Shaklan, S., "Phase-induced amplitude apodization complex mask coronagraphy (piaacmc) for large segmented apertures," Proc. SPIE (10400-200) (2017). Publication pending.

[2] Guyon, O., Martinache, F., Belikov, R., and Soummer, R., "High performance piaa coronagraphy with complex amplitude focal plane masks," The Astrophysical Journal Supplement Series 190(2), 220 (2010).

[3] Newman, K., Sirbu, D., Belikov, R., and Guyon, O., "Development of piaa complex mask coronagraphs for large aperture ground-based telescopes," Proc.SPIE 9912, 9912-9912-8 (2016).

[4] Newman, K., Conway, J., Belikov, R., and Guyon, O., "Focal plane phase masks for piaa: Design and manufacturing," Publications of the Astronomical Society of the Pacific 128(963), 055003 (2016).

[5] Kern, B., Guyon, O., Belikov, R., Wilson, D., Muller, R., Sidick, E., Balasubramanian, B., Krist, J., Poberezhskiy, I., and Tang, H., "Phase-induced amplitude apodization complex mask coronagraph mask fabrication, characterization, and modeling for wfirst-afta," Journal of Astronomical Telescopes, Instruments, and Systems 2(1), 011014 (2016).

[6] Jovanovic, N., Guyon, O., Lozi, J., Currie, T., Hagelberg, J., Norris, B., Singh, G., Pathak, P., Doughty, D., Goebel, S., Males, J., Kuhn, J., Serabyn, E., Tuthill, P., Schworer, G., Martinache, F., Kudo, T., Kawahara, H., Kotani, T., Ireland, M., Feger, T., Rains, A., Bento, J., Schwab, C., Coutts, D., Cvetojevic, N., Gross, S., Arriola, A., Lagadec, T., Kasdin, J., Groff, T., Mazin, B., Minowa, Y., Takato, N., Tamura, M., Takami, H., and Hayashi, M., "The scexao high contrast imager: transitioning from commissioning to science," Proc. SPIE 9909, 99090W-99090W-10 (2016).

[7] Guyon, O., "Piaacmcdesign." https://github.com/oguyon/PIAACMCdesign.

[8] Swanson, G. and Laboratory, L., [Binary optics technology: the theory and design of multi-level diffractive optical elements], Technical report (Lincoln Laboratory), Massachusetts Institute of Technology, Lincoln Laboratory (1989).

[9] Mack, C., [Introduction to Semiconductor Lithography], John Wiley and Sons, Ltd (2007).

[10] N'Diaye, M., Vigan, A., Dohlen, K., Sauvage, J.-F., Caillat, A., Costille, A., Girard, J. H. V., Beuzit, J.-L., Fusco, T., Blanchard, P., Le Merrer, J., Le Mignant, D., Madec, F., Moreaux, G., Mouillet, D., Puget, P., and Zins, G., "Calibration of quasi-static aberrations in exoplanet direct-imaging instruments with a zernike phase-mask sensor - ii. concept validation with zelda on vlt/sphere," A\&A 592, A79 (2016). 